\title{
APPLICATION OF CLIFFORD ALGEBRA TO ANALYSIS OF SPIN PROPERTIES OF SEMICONDUCTORS
}

\author{
A. Dargys \\ Semiconductor Physics Institute, A. Goštauto 11, LT-01108 Vilnius, Lithuania \\ E-mail: dargys@pfi.lt
}

Received 14 May 2009; revised 11 September 2009; accepted 15 September 2009

\begin{abstract}
The quantum mechanics is formulated in terms of state vectors in the Hilbert space and operators that act on these vectors. However, using the Clifford algebra an alternative formulation is possible, where multivectors in $n$-dimensional linear Euclidean space represent both the state of the system and quantum operators. In this report a short overview is presented on how the Clifford algebra can be used to investigate electron and hole spin properties in semiconductors. In particular, calculation of free electron and hole spin precession in cubic semiconductors with the help of the Clifford algebra is discussed.
\end{abstract}

Keywords: Clifford algebra, geometric algebra, cubic semiconductors, spintronics

PACS: 75.10.Jm, 85.75.-d, 72.25.Dc, 71.70.Ej

\section{Introduction}

The Clifford algebra $C l_{p, q}$ is a relatively new mathematical tool in theoretical physics to solve the problems in multidimensional spaces, especially when spinorial properties of the physical objects are important [1-3]. In the algebra $C l_{p, q}$, the sum of the indices $p+q$ gives the total dimension of the space while the individual indices, $p$ and $q$, designate the number of coordinates with positive and negative signature, respectively. Two of the algebras, $\mathrm{Cl}_{3,0}$ and $\mathrm{Cl}_{3,1}$, which are also called the geometric algebras $[2,4,5]$, are used to describe particles in 3D Euclidean and 4D Minkowski spaces. Up till now the Clifford algebra was applied to classical mechanics [6,7], Pauli and Dirac spinors in three and four dimensions [1,2], field theory [5], including classical electrodynamics [8-10], gravitation and cosmology [5], and recently in computer science [11]. The reader unfamiliar with the geometric algebra can refer to Hestenes paper [3] where one can find an accessible introduction, more references, as well as websites on the Clifford algebra.

In this report we shall overview the first attempts to adapt the Clifford algebra in the investigations of dynamical spin properties in semiconductors. In particular, we shall consider the precession of the conduction band electron and valence band hole spins in cubic semiconductors induced by spin-orbit interaction. As known, in the absence of such interaction the en- ergy bands in semiconductors are doubly degenerate (Kramers degeneracy [12]). The spin-orbit interaction splits the bands and brings about spin precession, the frequency of which depends on a considered energy band and charge carrier wave vector. Although the spin properties can be analysed in the standard manner within Hilbert space formalism [13-16], nonetheless, as we shall see the Clifford algebra may describe spin properties in a totally different and more elegant way. Since the considered subject is new to solid state physicists, in the first section of this article the required terminology for description of the Clifford algebras is introduced. Then, the spin properties of electrons within $\mathrm{Cl}_{3,0}$ algebra and hole properties within $\mathrm{Cl}_{5,0}$ and $\mathrm{Cl}_{4,1}$ algebras are considered. The starting point in all cases will be the Hamiltonian obtained by k.p method. At first, the spin dynamics of conduction band electron is considered and then the more difficult case of valence band holes is reviewed.

\section{Short introduction to Clifford algebras}

The Clifford algebra $C l_{p, q}$ is made up of $2^{p+q}$ elements, which usually are indicated by bold $\mathbf{e}$. Of these, there are $p+q$ basis vectors $\mathbf{e}_{i}$ which play the role of Cartesian coordinates. In $C_{3,0}$ the Euclidean space $\mathbb{R}^{3}$ is spanned by basis vectors $\mathbf{e}_{1}, \mathbf{e}_{2}$, and $\mathbf{e}_{3}$. Similarly, the five dimensional space $\mathbb{R}^{5}$ of $C l_{4,1}$ is spanned by basis vectors $\mathbf{e}_{1}, \ldots, \mathbf{e}_{5}$. The signature of the space is deter- 
mined by signs of $\mathbf{e}_{i}^{2}$. In $C l_{3,0}$, all $\mathbf{e}_{i}$ s satisfy $\mathbf{e}_{i}^{2}=+1$. However for $\mathrm{Cl}_{4,1}$ the square of the last basis vector is negative, $\mathbf{e}_{5}^{2}=-1$. The rest of elements in the Clifford algebra are made up of products of basis vectors. For short, the product $\mathbf{e}_{i} \mathbf{e}_{j}$ will be written as $\mathbf{e}_{12}$, similarly $\mathbf{e}_{i} \mathbf{e}_{j} \mathbf{e}_{k} \equiv \mathbf{e}_{i j k}$, etc. Thus, we will need $2^{3}=8$, or $2^{5}=32$ elements to describe the conduction band by $\mathrm{Cl}_{3,0}$ and the valence band by $\mathrm{Cl}_{4,1}$ algebra. One should pay attention that despite of this similarity the basis vectors are altogether different from the well-known unit vectors in $\mathbb{R}^{n}$ space, although the usual vectors, for example the wave vector $\mathbf{k}=\left(k_{x}, k_{y}, k_{z}\right)$, is mapped as $\mathbf{k} \rightarrow k_{x} \mathbf{e}_{1}+k_{y} \mathbf{e}_{2}+k_{z} \mathbf{e}_{3}$. Specifically, in contrast to the usual unit vectors the basis vectors in $C l_{p, q}$ do not commute. In fact, they anticommute, that is $\mathbf{e}_{i} \mathbf{e}_{j}=-\mathbf{e}_{j} \mathbf{e}_{i}$ if $i \neq j$. Both mentioned rules can be combined into the anticommutation relation

$$
\mathbf{e}_{i} \mathbf{e}_{j}+\mathbf{e}_{j} \mathbf{e}_{i}=2 \delta_{i j}
$$

where $\delta_{i j}$ is the Kronecker delta. Due to the property (1), the Clifford algebra elements make up a closed system, since the elements with repeated indices can be contracted, for example, $\mathbf{e}_{1232} \equiv \mathbf{e}_{1} \mathbf{e}_{2} \mathbf{e}_{3} \mathbf{e}_{2}=$ $-\mathbf{e}_{1} \mathbf{e}_{2} \mathbf{e}_{2} \mathbf{e}_{3}=-\mathbf{e}_{1} \mathbf{e}_{3}=-\mathbf{e}_{13}$. The largest product which cannot be reduced is called the pseudoscalar and written as $I$. Thus, in $\mathrm{Cl}_{3,0}$ algebra the pseudoscalar is $I=\mathbf{e}_{123}$, while in $C l_{5,0}$ and $C l_{4,1}$ algebras the pseudoscalar is $I=\mathbf{e}_{12345}$. The square of the pseudoscalar gives $I^{2}=1$ in $C l_{5,0}$, but it is $I^{2}=-1$ in $C l_{4,1}$. This is easy to prove by rearranging the basis vectors and using the property (1). Thus, in $C l_{4,1}$ the pseudoscalar $I$ performs the role of an imaginary unit.

The general vector $a$ in $\mathbb{R}^{n}$ can be expressed as a sum $a=\sum_{i} a_{i} \mathbf{e}_{i}$ of the elements of the same grade, where $a_{i}$ are real-valued components, $a_{i} \in \mathbb{R}$. Similarly, a general bivector can be written as a sum of elementary bivectors, $B=\sum_{i \neq j} b_{i j} \mathbf{e}_{i} \mathbf{e}_{j}$, where $b_{i j} \in \mathbb{R}$. In the Clifford algebra there exist the mixed grade elements as well. For example, one can add the vector to bivector to get the multivector $M=a+B$, or one can add the scalar to vector to get another multivector. The product of two multivectors of the same grade gives scalar, for example, the product of two vectors $a$ and $b=\sum_{i} b_{i} \mathbf{e}_{i}$ gives the scalar $a b=\sum_{i} a_{i} b_{i}$. However there also exists a mixed product, for example, of the vector $a$ with the bivector $B$. Such products may give higher or lower grade elements as compared to those of the factors.

A general multivector $M$ consists of a sum of multivectors of various grades:

$$
M=\sum_{i=0}\langle M\rangle_{i},
$$

where $\langle M\rangle_{0}$ is the scalar part of the multivector, $\langle M\rangle_{1}$ is the vector part, $\langle M\rangle_{2}$ is the bivector part, $\langle M\rangle_{3}$ is the trivector part, etc. The index $i$ is called the grade. As mentioned, the element of the highest grade is called the pseudoscalar. Sometimes it is convenient to express a high order grade element as a product of the pseudoscalar $I$ and lower grade element. For example, instead of $\mathbf{e}_{23}$ we may also write $I \mathbf{e}_{1}$, since in $C_{3,0}$ we have $\mathbf{e}_{23}=\mathbf{e}_{2} \mathbf{e}_{3}=\mathbf{e}_{2} \mathbf{e}_{3} \mathbf{e}_{1} \mathbf{e}_{1}=\mathbf{e}_{1} \mathbf{e}_{2} \mathbf{e}_{3} \mathbf{e}_{1}=I \mathbf{e}_{1}$. Similarly we can write $\mathbf{e}_{1} \mathbf{e}_{2}=I \mathbf{e}_{3}, \mathbf{e}_{3} \mathbf{e}_{1}=I \mathbf{e}_{2}$. All this can be expressed by the following table:

\begin{tabular}{cccc}
\hline$\langle M\rangle_{0}$ & $\langle M\rangle_{1}$ & $\langle M\rangle_{2}$ & $\langle M\rangle_{3}$ \\
\hline 1 & $\mathbf{e}_{i}$ & $I \mathbf{e}_{i}$ & $I$ \\
\hline 1 & 3 & 3 & 1 \\
\hline
\end{tabular}

where the first line shows the grade of a multivector, the second line shows a typical element, and the last line shows the number of elements of a given grade. Usually the index in the scalar part is omitted, and one writes $\langle M\rangle \equiv\langle M\rangle_{0}$. Similarly, the elements of $\mathrm{Cl}_{5,0}$ and $C l_{4,1}$ algebras can be grouped to the table

\begin{tabular}{cccccc}
\hline$\langle M\rangle_{0}$ & $\langle M\rangle_{1}$ & $\langle M\rangle_{2}$ & $\langle M\rangle_{3}$ & $\langle M\rangle_{4}$ & $\langle M\rangle_{5}$ \\
\hline 1 & $\mathbf{e}_{i}$ & $\mathbf{e}_{i j}$ & $I \mathbf{e}_{i j}$ & $I \mathbf{e}_{i}$ & $I$ \\
\hline 1 & 5 & 10 & 10 & 5 & 1 \\
\hline
\end{tabular}

One can multiply the multivectors. The product of multivectors $A, B$ and $C$ is associative, $A(B C)=$ $(A B) C=A B C$, however, it is noncommutative, $A B \neq B A$. Due to property (1), a given Clifford algebra $C l_{q, p}$ always remains closed under the multiplication.

There is a number of operations over the multivectors in the Clifford algebra which we shall make use of. For example, the multiplication by $I$ gives the dual multivector. From the above tables it is seen that all elements have their duals. Another important operation is the reversion that brings the basis vectors to reverse order. The reversion is denoted by tilde. For example, $\tilde{\mathbf{e}}_{12}=\mathbf{e}_{21}=-\mathbf{e}_{12}$. The reversion in the Clifford algebra plays similar role as the complex adjoint in the standard quantum mechanics. The typical operation is the product of multivector by its reverse. For example, in $C l_{5,0}$ this gives $\tilde{B} B=B \tilde{B}=\sum_{i \neq j} b_{i j}^{2}$, which represents the square of magnitude of a bivector.

One more important operation is the exponential of a multivector $A$ which is defined as a series $\mathrm{e}^{A}=$ $1+\frac{A}{1 !}+\frac{A^{2}}{2 !}+\frac{A^{3}}{3 !}+\cdots$. Typical situation where exponentials appear is the rotation of multivector in the multidimensional space. For a general multivector the exponential series cannot be summed up in a closed form. However, in specific situations the sum may be 
expressed through either trigonometric or hyperbolic functions. For example, if the multivector consists of a scalar $s$ and vector $v=v_{1} \mathbf{e}_{1}+v_{2} \mathbf{e}_{2}+v_{3} \mathbf{e}_{3}+v_{4} \mathbf{e}_{4}+v_{5} \mathbf{e}_{5}$, the exponential gives

$$
\mathrm{e}^{s+v}=\mathrm{e}^{s}\left(\sinh \sqrt{v \tilde{v}}+\frac{v}{\sqrt{v \tilde{v}}} \cosh \sqrt{v \tilde{v}}\right),
$$

where $v \tilde{v}=v_{1}^{2}+v_{2}^{2}+v_{3}^{2}+v_{4}^{2}+v_{5}^{2}$.

A useful property that will be frequently employed below is matrix representation of an element of the Clifford algebra. Concrete examples will be presented below in transforming the quantum mechanical operators to Clifford algebra representation.

\section{Conduction band}

In cubic semiconductors the conduction band is characterized by a $2 \times 2$ Hamiltonian that consists of an isotropic in the wave vector $\mathbf{k}$ part $\hat{H}_{0}$ and two spin-orbit (SO) related contributions, the Dresselhaus $\hat{H}_{\mathrm{D}}$ [17] and Rashba $\hat{H}_{\mathrm{R}}[18]$ ones: $\hat{H}=\hat{H}_{0}+\hat{H}_{\mathrm{D}}+$ $\hat{H}_{\mathrm{R}}$. In the case of free-space electrons, the formulation of quantum mechanics in terms of the Clifford algebra was described in Refs. [2,4]. In particular, the two-component spinor $|\psi\rangle$ here is placed in one-to-one correspondence with a 4-component multivector $\psi$ via the mapping

$$
|\psi\rangle=\left[\begin{array}{c}
a_{0}+\mathrm{i} a_{3} \\
-a_{2}+\mathrm{i} a_{1}
\end{array}\right] \longleftrightarrow \psi=a_{0}+\sum_{k=1}^{3} a_{k} I \mathbf{e}_{k},
$$

where all $a_{k}$ s are the real numbers. Thus, in $\mathrm{Cl}_{3,0}$ the spinor is represented by a sum of scalar and three bivector components. The ket-vector $|\psi\rangle$ and its Clifford algebra counterpart $\psi$ satisfy

$$
\langle\psi \mid \psi\rangle \longleftrightarrow\langle\tilde{\psi} \psi\rangle=\langle\psi \tilde{\psi}\rangle=a_{0}^{2}+a_{1}^{2}+a_{2}^{2}+a_{3}^{2} .
$$

From (4) it follows that the spin-up and spin-down states are related by rules $|\uparrow\rangle \longleftrightarrow 1$ and $|\downarrow\rangle \longleftrightarrow-I \sigma_{2}$. Also, additional replacement rules exist $[2,5,19,20]$

$$
\begin{aligned}
\mathrm{i} \partial|\psi\rangle / \partial t & \longleftrightarrow I \dot{\psi} \mathbf{e}_{3}, \\
\hat{\sigma}_{k}|\psi\rangle & \longleftrightarrow \mathbf{e}_{k} \psi \mathbf{e}_{3}, \\
\mathbf{k}^{2} \hat{\mathbf{1}}|\psi\rangle & \longleftrightarrow \mathbf{k}^{2} \psi
\end{aligned}
$$

that will be used to transform the Pauli-Schrödinger equation to the Clifford algebra equivalent expression. Here $\mathbf{k}=\left(k_{x}, k_{y}, k_{z}\right)$ is the electron wave vector referenced to crystallographic axes and $\hat{\sigma}_{k}$ is one of the Pauli matrices. To prove the rules (6) it is enough to resort to the mapping (4) and to remember that the multivectors do not commute. Then, in the case of the conduction band electron the required time-dependent PauliSchrödinger equation in the multivector notation can be reduced to the following compact form:

$$
\left.\dot{\psi}=-I \varepsilon_{0} \psi \mathbf{e}_{3}-I\left(\varepsilon_{1} \mathbf{e}_{1}+\varepsilon_{2} \mathbf{e}_{2}+\varepsilon_{3} \mathbf{e}_{3}\right) \psi\right),
$$

where $\varepsilon_{0}=\mathbf{k}^{2} /\left(2 m^{*}\right), \varepsilon_{1}=\gamma \chi_{1}+\alpha\left(k_{2} n_{3}-k_{3} n_{2}\right)$, and $\chi_{1}=k_{x}\left(k_{y}^{2}-k_{z}^{2}\right)$, and $n_{i}$ s are the components of the unit vector, which is perpendicular to heteroplane, in the Rashba Hamiltonian. The other components, $\varepsilon_{2}$ and $\varepsilon_{3}$, can be obtained from cyclic permutation of the indices. It should be noted that $\mathbf{e}_{3} \psi \neq \psi \mathbf{e}_{3}$. The solution of the differential equation (7) is the multivector

$$
\psi(t)=\psi_{i} \mathrm{e}^{-I\left(\varepsilon_{1} \mathbf{e}_{1}+\varepsilon_{2} \mathbf{e}_{2}+\varepsilon_{3} \mathbf{e}_{3}\right) t} \mathrm{e}^{-I \mathbf{e}_{3} \varepsilon_{0} t},
$$

where $\psi_{i}=\psi(0)$ is the initial multivector. $\psi(t)$ satisfies the normalization condition $\tilde{\psi}(t) \psi(t)=1$. Thus, the resulting multivector (8) can be factored as $\psi(t)=$ $\psi_{\mathrm{i}} \psi_{\text {so }} \psi_{0}$, where $\psi_{0}$ and $\psi_{\text {so }}$ are related, respectively, to degenerate part $\hat{H}_{0}$ and SO parts, $\hat{H}_{\mathrm{D}}$ and $\hat{H}_{\mathrm{R}}$, of the Hamiltonian:

$$
\psi_{0}=\cos \varepsilon_{0} t-I \sin \varepsilon_{0} t,
$$

$\psi_{\mathrm{SO}}=\cos |\varepsilon| t-\frac{1}{|\varepsilon|}\left(\varepsilon_{1} I \mathbf{e}_{1}+\varepsilon_{2} I \mathbf{e}_{2}+\varepsilon_{3} I \mathbf{e}_{3}\right) \sin |\varepsilon| t$.

The equations (8)-(10) show that there are two kinds of oscillations, those related with band degeneracy, $\varepsilon_{0}=$ $\mathbf{k}^{2} /\left(2 m^{*}\right)$ and those related with spin-orbit splitting of the degenerate bands, $\Delta E \equiv 2|\varepsilon|=2 \sqrt{\varepsilon \tilde{\varepsilon}}=$ $2 \sqrt{\varepsilon_{1}^{2}+\varepsilon_{2}^{2}+\varepsilon_{3}^{2}}$. The atomic unit system $e=\hbar=$ $m_{0}=1$ is used. Thus, the Clifford algebra shows in a very elegant way that the electron spin precession in cubic semiconductors can be described by three effective parameters only: $\varepsilon_{1}, \varepsilon_{2}$, and $\varepsilon_{3}$. In contrast, the solution of the same problem within the Hilbert space formalism appears to be rather messy [14]. In the latter case one is forced to shuttle between energy and $\hat{\sigma}_{z}$ representations of the Hamiltonian to describe the spin evolution in a mixed state.

The multivector $\psi$ allows one to calculate the spin precession dynamics in a straitghforward way. In $\mathrm{Cl}_{3,0}$ the electron spin polarization components are determined by mapping $[2,4,5]$

$$
\begin{aligned}
& \left\langle\psi\left|\hat{\sigma}_{k}\right| \psi\right\rangle \longleftrightarrow S_{k}=\boldsymbol{\sigma}_{k} \cdot \mathbf{S}, \quad \text { where } \\
& \mathbf{S}=\left\langle\psi \boldsymbol{\sigma}_{3} \tilde{\psi}\right\rangle_{1} .
\end{aligned}
$$

The average spin polarization components $S_{k}(k=$ $1,2,3$ or $x, y, z)$ depend on the initial multivector $\psi_{\mathrm{i}}$ 


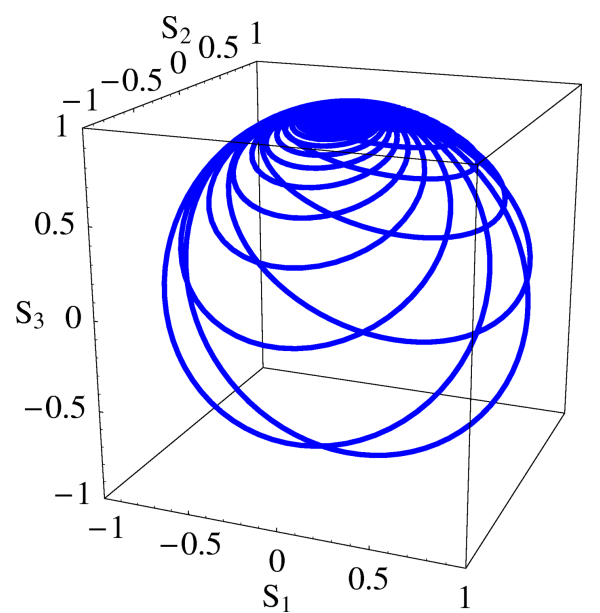

Fig. 1. Electron spin trajectories on the Bloch sphere. The energy $\varepsilon_{3}$ is varied and $\varepsilon_{1}=\varepsilon_{2}=0$. All precession trajectories run over the North pole.

and effective energies $\varepsilon_{1}, \varepsilon_{2}, \varepsilon_{3}$ only. Figure 1 illustrates spin polarization precession trajectories on the Bloch sphere calculated with equations (9)-(11). The initial condition $\psi_{\mathrm{i}}=1$, which corresponds to North pole on the sphere at $t=0$, was used.

It is well known that spin precession occurs only in mixed spin-up and -down states. Figure 1 shows that the solution (8) already incorporates such states. To have more precession trajectories it is enough to assume different multivector $\psi_{\mathrm{i}}$ at $t=0$. The spinor constructed in this way should be mapped by the rule (4). A possible choice may be

$$
\begin{aligned}
\psi_{\mathrm{i}}= & \cos \vartheta \cos \phi+I \mathbf{e}_{1} \sin \vartheta \sin \phi \\
& -I \mathbf{e}_{2} \sin \vartheta \cos \phi-I \mathbf{e}_{3} \cos \theta \sin \phi .
\end{aligned}
$$

Up and down spin states are recovered at $\vartheta=0, \phi=0$ and $\vartheta=\pi, \phi=0$, respectively. A more detailed exposition of application of the Clifford algebra $\mathrm{Cl}_{3,0}$, including quantum wells, and explicit analytical formulas for spin precession are presented in the article [21].

\section{Valence band}

\subsection{Choice of the Clifford algebra and matrix representation}

For conduction band, the unperturbed Hamiltonian $H_{0}$ coincides with the Pauli-Schrödinger one for electron with $\frac{1}{2}$ spin. As shown in Doran's PhD thesis [4] and book [2], the evolution of the respective spinor and multivector in this case can be described by the mapping (4). In the previous section this mapping was applied to conduction band electrons with SO interaction included. The valence band Hamiltonian has a different structure. It comes from $J=\frac{3}{2}$ manifold and can be constructed by k $\cdot \mathbf{p}$ perturbation method [22]. The resulting effective Hamiltonian is characterized by a number of empirical parameters depending of the number of bands included. In the following we shall consider the simplest valence band Hamiltonian that consists of the doubly degenerate light- and heavy-mass bands. In the spherical approximation this Hamiltonian reduces to [23]

$$
H_{0}=\frac{1}{2 m_{0}}\left[\left(\gamma_{1}+\frac{5}{2} \gamma_{2}\right) \mathbf{k}^{2}-2 \gamma_{2}(\mathbf{k} \cdot \mathbf{J})^{2}\right],
$$

where $\mathbf{J}=\left(J_{x}, J_{y}, J_{z}\right)$ is the vectorial $4 \times 4$ total angular momentum matrix, its components are given in [24]. $\gamma_{1}$ and $\gamma_{2}$ are the empirical band parameters. The matrix Hamiltonian (13) will be our starting point.

To map the valence band Hamiltonian onto one of the Clifford algebras we shall make use of the property that the basis vectors in the Clifford algebra can be represented by a set of complex valued matrices. For example, in the case of $\mathrm{Cl}_{3,0}$ there exists one-to-one correspondence between the basis vectors $\mathbf{e}_{1}, \mathbf{e}_{2}$, and $\mathbf{e}_{3}$ and the well-known three Pauli matrices. In particular, the Pauli matrices satisfy the same anticommutation relations as basis vectors (see Eq. (1)). The existence of isomorphism between basis vectors of the Clifford algebra and a set of special matrices allows one to express all elements of the algebra, including bivectors, pseudoscalars, etc, as respective products of the basis matrices. Matrix representation of the Clifford algebra may be also helpful in manipulating the multivectors, or in calculating the exponentials of the multivectors. The existence of an isomorphism between the matrix representation and a particular Clifford algebra allows an easy transition from matrix formulation of the quantum mechanics to its Clifford analogues. This property will be used below.

Now, we shall transform the valence band Hamiltonian in matrix representation (13) to Clifford algebra representation. To select a suitable one it is convenient to address to a table of all possible matrix representations that are usually given along with the 8-fold periodicity theorem of real Clifford algebras [1]. From this table it follows that the Clifford vectors in $\mathbb{R}^{5}$ are isomorphic to ${ }^{2} \mathbb{H}(2)$, i. e. to double quaternionic $2 \times 2$ matrices $\mathbb{H}(2)$. However, this representation is not suitable for our purpose since our starting matrices are complex rather than quaternionic valued. There are three complex candidate algebras, namely, $\mathrm{Cl}_{0,5}, \mathrm{Cl}_{2,3}$, and $\mathrm{Cl}_{4,1}$, which are isomorphic to complex matrices $\mathbb{C}(4)$. Their 
basis vectors abide in 5D Euclidean space $\mathbb{R}^{5}$ and have the following signatures: $(-----),(++---)$, and $(++++-)$. Nevertheless, if a suitable isomorphism is absent, then, with a due care, one can also resort to homomorphism between Clifford algebra and its matrix counterpart having an appropriate symmetry. For example, the matrix representation that corresponds to $\mathrm{SO}(5)$ group and which is homomorphic to $\mathrm{Cl}_{5,0}$ algebra can be used to map the Hamiltonian (13) to Clifford algebra representation [25,26]. Below we shall briefly discuss two representations having $\mathrm{SO}(5)$ and $\mathrm{SO}(4,1)$ symmetry that are associated, respectively, with the Clifford algebras $C l_{5,0}$ and $C l_{4,1}$. The application of $\mathrm{Cl}_{2,3}$ algebra still remains uninvestigated.

\section{2. $\mathrm{Cl}_{5,0}$ algebra}

The required $4 \times 4$ matrices that have totally positive signature, $\mathbf{e}_{1}^{2}=\mathbf{e}_{2}^{2}=\mathbf{e}_{3}^{2}=\mathbf{e}_{4}^{2}=\mathbf{e}_{5}^{2}=1$, are [25]

$$
\begin{aligned}
& \mathbf{e}_{1} \rightarrow \Gamma_{1}=\left[\begin{array}{cc}
\hat{\sigma}_{y} & 0 \\
0 & -\hat{\sigma}_{y}
\end{array}\right], \\
& \mathbf{e}_{2} \rightarrow \Gamma_{2}=\left[\begin{array}{cc}
\hat{\sigma}_{x} & 0 \\
0 & -\hat{\sigma}_{x}
\end{array}\right], \\
& \mathbf{e}_{3} \rightarrow \Gamma_{3}=\mathrm{i}\left[\begin{array}{cc}
0 & -1 \\
1 & 0
\end{array}\right], \\
& \mathbf{e}_{4} \rightarrow \Gamma_{4}=\left[\begin{array}{ll}
0 & 1 \\
1 & 0
\end{array}\right], \\
& \mathbf{e}_{5} \rightarrow \Gamma_{5}=\left[\begin{array}{cc}
\hat{\sigma}_{z} & 0 \\
0 & -\hat{\sigma}_{z}
\end{array}\right] .
\end{aligned}
$$

Using these matrices the Hamiltonian (13) can be rewritten in the Clifford algebra as

$$
H_{0}=\frac{\gamma_{1} k^{2}}{2 m_{0}}+\frac{\gamma_{2} k^{2}}{m_{0}} \sum_{n=1}^{5} d_{n} \mathbf{e}_{n},
$$

where $k^{2}=k_{x}^{2}+k_{y}^{2}+k_{z}^{2}$ and $d_{n}$ are the real-valued projections on the respective basis vectors $\mathbf{e}_{i}$ in $\mathbb{R}^{5}$ space:

$$
\begin{aligned}
& d_{1}=-\sqrt{3} k_{y} k_{z} / k^{2}, \quad d_{2}=-\sqrt{3} k_{x} k_{z} / k^{2}, \\
& d_{3}=-\sqrt{3} k_{x} k_{y} / k^{2}, \quad d_{4}=-\frac{\sqrt{3}}{2}\left(k_{x}^{2}-k_{y}^{2}\right) / k^{2}, \\
& d_{5}=-\frac{1}{2}\left(2 k_{z}^{2}-k_{x}^{2}-k_{y}^{2}\right) / k^{2} .
\end{aligned}
$$

From (19) it is seen that the Hamiltonian is made up of the scalar and vector in $\mathbb{R}^{5}$. Since the squares of projec- tions give $d_{1}^{2}+d_{2}^{2}+d_{3}^{2}+d_{4}^{2}+d_{5}^{2}=1$ for all possible directions and magnitudes of $\mathbf{k}$, the vector $d$ represents the radius of a $4 \mathrm{D}$ sphere in $\mathbb{R}^{5}$. This suggests that the Hamiltonian can be diagonalized by an appropriate rotation of the vector $d=\sum_{n=1}^{5} d_{n} \mathbf{e}_{n}$ in $\mathbb{R}^{5}$ Euclidean space. Thus, we can find the eigenvalues of the Hamiltonian and perform spin dynamics calculations without using the Hilbert space at all. How this can be achieved with the help of rotors $R$ defined in the Clifford algebra the reader should refer to paper [27]. Here we shall present only the final result:

$\mathcal{H}_{d}=\tilde{R} H R=\frac{k^{2}}{2 m_{0}}\left(\gamma_{1}+2 \gamma_{2} \mathbf{e}_{5}\right) \rightarrow\left[\begin{array}{cccc}\varepsilon_{\mathrm{L}} & 0 & 0 & 0 \\ 0 & \varepsilon_{\mathrm{H}} & 0 & 0 \\ 0 & 0 & \varepsilon_{\mathrm{H}} & 0 \\ 0 & 0 & 0 & \varepsilon_{\mathrm{L}}\end{array}\right]$,

where $\varepsilon_{\mathrm{H}, \mathrm{L}}=\left(\gamma_{1} \pm 2 \gamma_{2}\right) k^{2} /\left(2 m_{0}\right)$ are the dispersions of heavy- and light-mass holes. The matrix representation in (21) was obtained with the mapping (18). From this short discussion it follows that in the Clifford algebra one can calculate heavy- and light-hole energies and spin polarizations by an appropriate rotation of the multivector in the five dimensional Euclidean space. For more details the reader is directed to paper [27].

\section{3. $\mathrm{Cl}_{4,1}$ algebra}

The matrices that represent basis vectors in $\mathbb{R}^{5}$ now are

$$
\begin{aligned}
& \mathbf{e}_{1} \rightarrow \Gamma_{1}=\mathrm{i}\left[\begin{array}{cc}
0 & \hat{\sigma}_{x} \\
-\hat{\sigma}_{x} & 0
\end{array}\right], \\
& \mathbf{e}_{2} \rightarrow \Gamma_{2}=\mathrm{i}\left[\begin{array}{cc}
0 & \hat{\sigma}_{y} \\
-\hat{\sigma}_{y} & 0
\end{array}\right], \\
& \mathbf{e}_{3} \rightarrow \Gamma_{3}=\mathrm{i}\left[\begin{array}{cc}
0 & \hat{\sigma}_{z} \\
-\hat{\sigma}_{z} & 0
\end{array}\right], \\
& \mathbf{e}_{4} \rightarrow \Gamma_{4}=\left[\begin{array}{ll}
0 & 1 \\
1 & 0
\end{array}\right], \\
& \mathbf{e}_{5} \rightarrow \Gamma_{5}=\mathrm{i}\left[\begin{array}{cc}
-1 & 0 \\
0 & 1
\end{array}\right] .
\end{aligned}
$$

These matrices as well as basis vectors have the signature $\mathbf{e}_{1}^{2}=\mathbf{e}_{2}^{2}=\mathbf{e}_{3}^{2}=\mathbf{e}_{4}^{2}=1$ and $\mathbf{e}_{5}^{2}=-1$. As a result, the square of the pseudoscalar is negative, $I^{2}=-1$. 
The Hamiltonian (13) in this algebra assumes the following form:

$$
\begin{aligned}
& H_{0}=\frac{\gamma_{1} k^{2}}{m_{0}} D_{0}+\frac{2 \gamma_{2} k^{2}}{m_{0}} \\
& \times\left(D_{1} \mathbf{e}_{4}+D_{2} \mathbf{e}_{45}+D_{3} \mathbf{e}_{215}+D_{4} \mathbf{e}_{325}+D_{5} \mathbf{e}_{135}\right),
\end{aligned}
$$

where the coefficients are $D_{0}=1 / 2, D_{1}=-d_{4} / 2$, $D_{2}=d_{3} / 2, D_{3}=-d_{5} / 2, D_{4}=-d_{1} / 2, D_{5}=d_{2} / 2$. Contrary to the expression (19), now the elements of $H_{0}$ in (27) have mixed grades. Therefore, the vector rotation in 5D Euclidean space in finding the eigenvalues is of little use. It can be shown that the diagonalized form of $H_{0}$ now is

$$
\mathcal{H}_{0}=\frac{\varepsilon_{\mathrm{H}}+\varepsilon_{\mathrm{L}}}{2}+\frac{\varepsilon_{\mathrm{H}}-\varepsilon_{\mathrm{L}}}{2} I \mathbf{e}_{5} \rightarrow\left[\begin{array}{cccc}
\varepsilon_{\mathrm{H}} & 0 & 0 & 0 \\
0 & \varepsilon_{\mathrm{H}} & 0 & 0 \\
0 & 0 & \varepsilon_{\mathrm{L}} & 0 \\
0 & 0 & 0 & \varepsilon_{\mathrm{L}}
\end{array}\right]
$$

and consists of a sum of the scalar and pseudoscalar.

The Hamiltonian (28) represents degenerate energy bands with no SO splitting and, therefore, with no spin precession dynamics. (In the following the operators written in the representation (28) will be in the calligraphic font.) To work on further, in the description of spin dynamics the SO interaction must be included. The needed SO interaction Hamiltonian mapped onto $C l_{4,1}$ algebra is [28]

$$
\begin{aligned}
& H_{1}= \\
& \frac{c}{2}\left(\sqrt{3} k_{x} \mathbf{e}_{1}+\sqrt{3} k_{y} \mathbf{e}_{2}+2 k_{z} \mathbf{e}_{35}+k_{x} \mathbf{e}_{245}-k_{y} \mathbf{e}_{415}\right),
\end{aligned}
$$

where $c$ is the SO splitting strength. The spin dynamics is described by similar differential equation as (7) except that now the pseudoscalar is $I \equiv \mathbf{e}_{12345}$ and the expression in the square brackets in (7) should be replaced by the total Hamiltonian $H=H_{0}+H_{1}$ written in $\mathrm{Cl}_{4,1}$. As previously, the solution can be written immediately: $\psi(t)=\mathrm{e}^{-I H t}$. Thus, the problem reduces to calculation of the exponential of the mixed multivector which should give all possible spin precession frequencies (compare Eqs. (9)-(10)). However, now the resulting series after the expansion of the exponent cannot be summed up in a closed form. Why? This can be seen from the eigenvalues of the Hamiltonian $H$. After the inclusion of the SO interaction all energy levels become nondegenerate. The spectrum of such Hamiltonian is described by the fourth order algebraic equation, the roots of which in general cannot be calculated explicitly. Thus, to find an analytical solution for all pos- sible spin precession frequencies one is forced to rely on approximate methods, similarly as it is in the treatment of problem within standard quantum mechanics. In the following we shall briefly discuss how this can be achieved in the Clifford algebra formalism.

At first, we shall assume that all operators have been rewritten in the energy representation of the degenerate Hamiltonian (28). Then, one can exploit the fact that the SO splitting of bands is small compared to degenerate light- or heavy-hole energy. To proceed with the perturbation theory we introduce the projection operators onto heavy- and light-mass energy bands:

$$
\mathcal{P}_{\mathrm{H}}=\frac{1}{2}\left(1+I \mathbf{e}_{5}\right), \quad \mathcal{P}_{\mathrm{L}}=\frac{1}{2}\left(1-I \mathbf{e}_{5}\right) .
$$

It is easy to check that these operators are idempotent, $\mathcal{P}_{\mathrm{H}}^{2}=\mathcal{P}_{\mathrm{H}}$ and $\mathcal{P}_{\mathrm{L}}^{2}=\mathcal{P}_{\mathrm{L}}$, and orthogonal, $\mathcal{P}_{\mathrm{L}} \mathcal{P}_{\mathrm{H}}=$ 0 . The degenerate Hamiltonian (28) remains invariant to action of the projection operators. To see where the perturbation theory comes in let us apply the projection operators to an arbitrary operator $\mathcal{A}$,

$$
\begin{aligned}
& \left(\mathcal{P}_{\mathrm{H}}+\mathcal{P}_{\mathrm{L}}\right) \mathcal{A}\left(\mathcal{P}_{\mathrm{H}}+\mathcal{P}_{\mathrm{L}}\right)= \\
& \mathcal{P}_{\mathrm{H}} \mathcal{A} \mathcal{P}_{\mathrm{H}}+\mathcal{P}_{\mathrm{L}} \mathcal{A} \mathcal{P}_{\mathrm{L}}+\mathcal{P}_{\mathrm{H}} \mathcal{A} \mathcal{P}_{\mathrm{L}}+\mathcal{P}_{\mathrm{L}} \mathcal{A} \mathcal{P}_{\mathrm{H}}
\end{aligned}
$$

The above operator equation is exact. In the following we shall neglect the cross terms, $\mathcal{P}_{\mathrm{H}} \mathcal{A} \mathcal{P}_{\mathrm{L}}$ and $\mathcal{P}_{\mathrm{L}} \mathcal{A} \mathcal{P}_{\mathrm{H}}$, which should be small if light- and heavy-mass bands are far apart. Thus, in our perturbation theory the SO interaction couples the terms in the degenerate band manifolds only. The calculations along these lines give us the heavy- and light-mass band Hamiltonians:

$$
\begin{aligned}
& \mathcal{H}_{H}=\frac{\gamma_{1}-2 \gamma_{2}}{2 m} k^{2}-\frac{3 \sqrt{3}}{2} c k\left[\left(\cos \theta \sin ^{2} \theta+\sin \theta\right.\right. \\
& \left.\left.\times \cos ^{2} \theta \mathbf{e}_{2}\right) \cos 2 \varphi-\frac{3+\cos 2 \theta}{4} \sin \theta \sin 2 \varphi \mathbf{e}_{3}\right], \\
& \mathcal{H}_{L}=\frac{\gamma_{1}+2 \gamma_{2}}{2 m} k^{2}-\frac{\sqrt{3}}{2} c k\left[\left(\cos \theta \sin ^{2} \theta+\sin \theta\right.\right. \\
& \left.\left.\times \cos ^{2} \theta \mathbf{e}_{2}\right) \cos 2 \varphi+\frac{1+3 \cos 2 \theta}{4} \sin \theta \sin 2 \varphi \mathbf{e}_{3}\right],
\end{aligned}
$$

where $\theta$ and $\phi$ are polar angles of the wave vector $\mathbf{k}$. It is seen that the SO splitting vanishes if $\theta=0$, i. e. when the hole wave vector is $\mathbf{k} \|$ [001], or the vector points towards equivalent crystallographic directions. This is a well-known fact for cubic semiconductors. The largest SO splitting is at $\mathbf{k} \|[011]$. The structure of the multivectors (32) and (33) suggests (compare it with the 


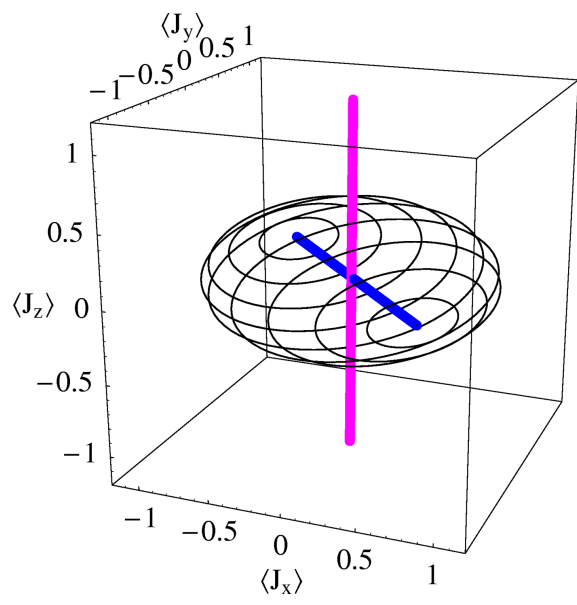

Fig. 2. Spin trajectories of the light-mass hole for various parameter $\vartheta$ values. The hole wave vector has polar angles $\theta=0.01, \varphi=$ $\pi / 4$ and is drawn by vertical line. The horizontal line is the spin precession axis.

multivector (19), where all basis vectors appear in the Hamiltonian) that the Clifford algebra that describes hole spin dynamics in decoupled heavy-light band approximation can be reduced to lower dimensional Clifford algebra. Indeed, it can be shown rigorously that in this approximation the total angular momentum operator which determines the precession of the spin polarization vector also reduces to lower Clifford algebra, namely, to $\mathrm{Cl}_{3,0}$ algebra. The latter, as discussed earlier, describes conduction band electron spin precession when electron is in a superposition of nearly degenerate twofold energy band. However, now the elements of Hamiltonians and respective total angular momentum operators are different from those for electron. As a result, hole spin procession trajectories will be different, too.

Figure 2 illustrates the precession character of total angular momentum $\langle\mathbf{J}\rangle$ of light-mass hole that was calculated within the framework of the Clifford algebra. In this approximation the hole spin $\langle\mathbf{S}\rangle$ precession coincides with that for $\langle\mathbf{J}\rangle$ but with the polarization vector lengths reduced by factor $2 / 3$. We see that now $\langle\mathbf{J}\rangle$ and $\langle\mathbf{S}\rangle$ precession trajectories are ellipses rather than circles as was the case for the conduction band electrons. This conclusion is consistent with our earlier calculations done within a standard quantum mechanical formalism $[16,29]$. It can be shown that the precession axis around which hole spin rotates is perpendicular to the hole wave vector $\mathbf{k}$ for all directions of $\mathbf{k}$. Similar calculations yield that the heavy-hole precession trajectories for $\langle\mathbf{J}\rangle$ reduce to vibrations along the straight lines of length $3 / 2$, the orientation of which in spin space depends on the direction of $\mathbf{k}$. This is also in agreement with earlier calculations [16, 29].

\section{Conclusions}

This short overview demonstrates that the Clifford algebra can be used in the analysis of electron and hole properties in semiconductors with some advantage over the standard methods. In the standard quantum mechanics one works in the Hilbert space spanned by linearly independent state vectors that may be represented by columns, and where the quantum mechanical operators (matrices) are acting on the state vectors. In the Clifford algebra one works in the Euclidean space $\mathbb{R}^{n}$ and both the spinors and operators are treated on the same footing, and as a result the formal difference between them vanishes. In case of electrons in cubic semiconductors, the conduction bands can be treated within $\mathrm{Cl}_{3,0}$ Clifford algebra. The spin precession trajectories in this case can be very easily calculated, in fact, in few strokes. There is no need to construct unitary matrices and to shuttle between $\hat{\sigma}_{z}$ and energy representations. The application of the multivector formalism to nanostructures where the space quantization plays an important role is uninvestigated as yet, although the multivector calculus needed for this purpose is already worked out [30].

In the case of the valence bands the situation is more complicated. There is a number of Clifford algebras that can be applied for this purpose. Two of these, $\mathrm{Cl}_{5,0}$ and $C l_{4,1}$, have been addressed here. At this stage of the investigation it is difficult to indicate whichever of algebras will be superior. However, preliminary calculations show that spin precession equations for valence band holes can be found in a more straightforward way with the help of the $C l_{4,1}$ Clifford algebra.

\section{Acknowledgement}

The work was partly supported by the Lithuanian State Science and Studies Foundation under contract C07004.

\section{References}

[1] P. Lounesto, Clifford Algebras and Spinors (Cambridge University Press, Cambridge, 1997).

[2] C. Doran and A. Lasenby, Geometric Algebra for Physicists (Cambridge University Press, Cambridge, 2003).

[3] D. Hestenes, Oersted medal lecture 2002: Reforming the mathematical language of physics, Am. J. Phys. 71(2), 104-121 (2003). 
[4] C. Doran, Geometric Algebra and its Application to Mathematical Physics, PhD thesis (University of Cambridge, 1994).

[5] C. Doran, A. Lasenby, S. Gull, S. Somaroo, and A. Challinor, Spacetime algebra and electron physics, arXiv:quant-ph/0509178v1, 1-93 (2005).

[6] D. Hestenes, New Foundations for Classical Mechanics (Reidel, Boston, 1985).

[7] T.G. Vold, An introduction to geometric algebra with an application in rigid body mechanics, Am. J. Phys. 61(6), 491-504 (1993).

[8] B. Jancewicz, Multivectors and Clifford Algebra in Electrodynamics (World Scientific, Singapore, 1988).

[9] W. Baylis, Electrodynamics: A Modern Geometric Approach (Birkhäuser, Boston, 1999).

[10] T.G. Vold, An introduction to geometric calculus and its application to electrodynamics, Am. J. Phys. 61(6), 505-513 (1993).

[11] L. Dorst, D. Fontijne, and S. Mann, Geometric Algebra for Computer Science (Morgan Kauffman Publishers, 2007-2008).

[12] C. Kittel, Quantum Theory of Solids (John Wiley and Sons, New York, 1963).

[13] A. Dargys, Hole spin surfaces in $\mathrm{A}_{3} \mathrm{~B}_{5}$ semiconductors, Phys. Status Solidi B 241(13), 2954-2961 (2004).

[14] A. Dargys, Free electron spin in cubic semiconductors, Lithuanian J. Phys. 46(1), 27-31 (2006).

[15] A. Dargys, Precession trajectories of the hole spin in zinc-blende semiconductors, Solid-State Electron. 51(1), 93-100 (2007).

[16] A. Dargys, Bloch sphere, spin surfaces and spin precession in quantum wells, in Spintronics: Materials, Applications and Devices, eds. G.C. Lombardi and G.E. Bianchi (NOVA Publishers, New York, 2009).

[17] G. Dresselhaus, Spin-orbit coupling effects in zinc blende structures, Phys. Rev. 100(2), 580-586 (1955).

[18] Yu.A. Bychkov and E.I. Rashba, Oscillatory effects and the magnetic susceptibility of carriers in inversion layers, J. Phys. C 17, 6039-6045 (1984).

[19] C. Doran, A. Lasenby, S. Gull, S. Somaroo, and A. Challinor, Spacetime algebra and electron physics, Adv. Imag. Electron Phys. 95, 271-365 (1996).

[20] D. Hestenes, Spin and uncertainty in the interpretation of quantum mechanics, Am. J. Phys. 47(5), 399-415 (1979).

[21] A. Dargys, Analysis of electron spin in semiconductors using geometric algebra, Phys. Scripta 79(5), 0557021-6 (2009).

[22] P. Yu and M. Cardona, Fundamentals of Semiconductors (Springer-Verlag, Berlin-Heidelberg-New York, 2005) Ch. 2.

[23] J.M. Luttinger and W. Kohn, Motion of electrons and holes in perturbed periodic fields, Phys. Rev. 97(4), 869-883 (1955).

[24] J.M. Luttinger, Quantum theory of cyclotron resonances in semiconductors: General theory, Phys. Rev. 102(4), 1030-1041 (1956).

[25] E. Demler and S.C. Zhang, Non-Abelian holonomy of BCS and SDW quasiparticles, Ann. Phys. 271, 83-119 (1999).

[26] S. Murakami, N. Nagaosa, and S.-C. Zhang, SU(2) non-Abelian holonomy and dissipationless spin current in semiconductors, Phys. Rev. B 69(23), 235206-1-14 (2004).

[27] A. Dargys, Valence band of cubic semiconductors from viewpoint of Clifford algebra, Acta Phys. Pol. A 116(2), 226-231 (2009).

[28] A. Dargys, Application of Clifford algebra to analysis of spin properties of semiconductors, accepted for publication in Phys. Scripta.

[29] A. Dargys, Hole spin surfaces in $A_{3} B_{5}$ semiconductors, Phys. Status Solidi B 241(13), 2954-2961 (2004).

[30] D. Hestenes and G. Sobczyk, Clifford Algebra to Geometric Calculus (Reidel, Boston, 1984).

\title{
CLIFFORDO ALGEBROS TAIKYMAS SUKINIŲ SAVYBIŲ ANALIZĖJE PUSLAIDININKIAMS
}

\author{
A. Dargys \\ Puslaidininkiu fizikos institutas, Vilnius, Lietuva
}

\begin{abstract}
Santrauka
Kvantinè mechanika suformuluota matematinès Hilberto erdvès sąvokomis, kur svarbų vaidmeni turi būsenų vektoriai bei operatoriai, kurie vienus Hilberto erdvès vektorius perveda i kitus. Tačiau yra žinoma alternatyvi kvantinės mechanikos formuluotė [1,2], kurioje dalelès būsena bei fizikinius dydžius aprašantys operatoriai yra nusakomi Cliffordo algebros sąvokomis, būtent, per daugiamatės Euklido erdvès vektorius ir multivektorius. Pastarieji yra sukonstruoti iš $n$-matès tiesinès Euklido erdvès bazinių vektorių visu galimų sandaugų. Būdinga tokių bazinių vektorių savybė ta, kad
\end{abstract}

jie tarpusavyje antikomutuoja, todèl Cliffordo algebros multivektoriais galima aprašyti spinorius bei jų dinamiką išoriniuose laukuose. Multivektoriaus projekcijos, kurios nusako matuojamus dydžius, yra realūs (o ne kompleksiniai, kaip Hilberto erdvès atveju) skaičiai. Cliffordo algebroje dalelès būsena ir operatoriai yra lygiavertès sąvokos ir nusakomos panašiai. Straipsnyje trumpai apžvelgta, kaip galima pritaikyti Cliffordo algebros formalizmą sukinių savybiu analizeje. Aptarta kubinès simetrijos puslaidininkio laidumo juostos elektrono ir valentinès juostos skylès sukinio precesija bei kaip ją būtų galima apskaičiuoti naudojant Cliffordo algebrą. 